\title{
Vom Räsonieren zum Entscheiden
}

\section{Die wissenschaftliche Diskussion zur Abschätzung der Anwendungs- möglichkeiten von Schlüsseltechnologien ist für die Planung in Politik und Wirt- schaft meist zu abstrakt. Eine Alternative für Entscheidungsträger ist die Tech- nologiefrüherkennung. Für Unternehmen gibt sie Hilfestellung zur Strategieent- wicklung und bietet die Grundlage für begleitende innovationspolitische Maß- nahmen. Die Politik kann Technologiefrüherkennung bei der Zusammenführung von gesellschaftlichem und wirtschaftlichem Bedarf sowie technisch-wissen- schaftlichem Angebot unterstützen.}

Von Axel Zweck

G emäß einer VDI-Studie zeichnen sich Schlüsseltechnologien durch folgende Charakteristika aus (1). Sie

- sind ein Schlüssel zu wirtschaftlichem Erfolg,

- erschließen neue Märkte und Wachstumsfelder,

- sind für eine Industriegesellschaft unverzichtbar,

- setzen sich in relativ kurzer Zeit durch,

- sind relativ einfach beherrschbar,

- sind breit verfügbar,

- bringen erhebliche soziale Veränderungen mit sich und

- müssen deshalb von der Gesellschaft 'verkraftet' werden.

Eine aufschlussreiche Übersicht, welche Technologien in den letzten Jahrzehnten mit diesen Eigenschaften gesehen wurden, ergibt sich aus einer Gegenüberstellung der als zentral gesehenen Technologiefelder aus verschieden Studien zu 'Schlüsseltechnologien' wie sie Abbildung 1 bietet. Die Übersicht zeigt, dass alle Felder mehrfach erwähnt wurden, einige in allen Studien. Wenn aber wichtige prospektive Studien über den Zeitraum eines Jahrzehntes zu einem mehr oder weniger unveränderten Ergebnis bezüglich künftiger Schlüsselfelder gelangen, ist die damit verbundene Aussagekraft gering. Für strategische Planungen in Wirtschaft wie Politik reduziert sich die Aussagekraft derartiger Darstellungen auf ein allenfalls strukturierendes Element. Eine Antwort auf die Frage, welche Technologien Schlüsseltechnologien sind, ist auf diesem hohen Abstraktionsgrad wenig hilfreich.

Als strategische Unterstützung von forschungsund technologiepolitischen Entscheidungen in Wirtschaft wie Politik zur Bestimmung zukunfts- relevanter und gesellschaftsbestimmender Technologien, sind differenziertere Betrachtungen auf konkreterem Niveau erforderlich. Wie aber können konkrete Hinweise auf zukunftsrelevante Technologien erschlossen werden? Einen Weg, subjektive Bewertungen weitgehend durch eine transparente, nachvollziehbare und an offenen Kriterien orientierte Identifikation und Bewertung zu ersetzen, bietet die Technologiefrüherkennung. Bei der Technologiefrüherkennung geht es um die Suche nach einer Schnittmenge zwischen wissenschaftlich Interessantem, technisch Beherrschbarem und an gesellschaftlich und wirtschaftlich Orientiertem. Technologiefrüherkennung ist eine vormarktliche Filterung von Innovationen auf Basis eines spezifischen und bedarfsorientierten Kriterienrasters. Ihr übergeordnetes Ziel ist die strategische Optimierung des Einsatzes von FuE-Ressourcen durch Fokussieren auf die Technologien mit größter Hebelwirkung bezüglich der herangezogenen Kriterien. Die Wahl der Bewertungskriterien hängt unter anderem davon ab, ob die zu identifizierenden Technologien für öffentliche Maßnahmen, für eine bestimmte Branche oder ein spezielles Unternehmensportfolio von Interesse sein sollen. Beim Ermitteln zukunftsrelevanter Technologiefelder müssen Einflussfaktoren möglichst umfassend einbezogen werden.

Die Technologiefrüherkennung lässt sich vereinfachend in drei Teilziele gliedern, auch wenn sie in der Praxis nicht immer leicht abgrenzbar sind. Erster Schritt ist das Suchen und Erkennen technisch-wissenschaftlicher Innovationen. Sie ergeben sich vor allem durch

- Entdecken eines neuen Grundeffektes (Hochtemperatursupraleitung)

- Weiterentwicklungen eines bekannten Ansatzes (Bionik)

- Heranziehen einer neuen Betrachtungsperspektive (nichtlineare Dynamik)

- die Suche nach Problemlösungen (neue Kühltechniken zur Ressourcenschonung)

Alle Aktivitäten im Rahmen des ersten Schrittes werden als Identifikation bezeichnet. Da sich derartige Innovationen und künftige Anwendungen weder von selbst ankündigen, noch immer sofort und offensichtlich als solche erkannt wer-den, geht es hier um einen aktiven Suchprozess.

\begin{tabular}{|c|c|c|c|}
\hline \multicolumn{4}{|c|}{ Abbildung 1: Technologiebezogene Zukunftsstudien aus Deutschland } \\
\hline $\begin{array}{c}\text { Schlüsseltechnologien } \\
\text { Schlösseltechn. } \\
\text { '87 }\end{array}$ & $\begin{array}{l}\text { Entwicklungslinien } \\
\text { Techno. 21. Jhdt. } \\
\text { '92 }\end{array}$ & $\begin{array}{c}\text { Fachgebiete } \\
\text { Delphi } \\
' 93\end{array}$ & $\begin{array}{l}\text { Themenfelder } \\
\text { Delphi } \\
\text { '98 }\end{array}$ \\
\hline Informationstechnik & Mikroelektronik & $\overline{l u K}$ & IUK \\
\hline Mikroelektronik & Software und Simulation & Elektronik & \\
\hline Software & Molekularelektronik & & \\
\hline & Nanotechnologie & & \\
\hline Neue Materialien & Neue Werkstoffe & Neue Werkstoffe & Chemie \& Werkstoffe \\
\hline Kunststoffe/Metall & & & \\
\hline Biotechnologie & Zell- Biotechnologie & Biowissenschaften & Gesundheit \& Lebensprozesse \\
\hline Pharmakologie & & Medizintechnik & Landwirtschaft \& Ernährung \\
\hline Laser & Photonik & & \\
\hline Weltraumtechnik & & Raumfahrt & Raumfahrt \\
\hline Tiefseetechnik & & Meeres- \& Geotechnik & \\
\hline & & Rohstofftechnik & \\
\hline Energie & & Energie & Energie \& Rohstoffe \\
\hline Supraleitung & Produktion \& Management & Kernphysik & \\
\hline & & Produktion & Produktion \& Management \\
\hline & & Verkehrstechnik & Mobilität \& Verkehr \\
\hline & & Umwelttechnik & Umwelt \& Natur \\
\hline & Bautechnik & Bauen \& Wohnen & \\
\hline
\end{tabular}

Quelle: siehe Anmerkung (2) 
Nach möglichst frühzeitigem Erkennen und Abgrenzen eines aussichtsreichen Technologiegebietes ist ein Einordnen in den Kontext der technisch-wirtschaftlichen Gesamtentwicklung erforderlich. Hierbei geht es vor allem um das Bewerten und Einschätzen von

- potentiellen Anwendungsfeldern

- zeitlichen Realisierungsperspektiven

- dem notwendigen Aufwand bis zur Anwendungspraxis

- den Auswirkungen auf andere Technologien und Entwicklungslinien.

Die Kriterien der Bewertung, wie sie im Rahmen der Technologiefrüherkennung im Auftrag des Bundesministeriums für Bildung und Forschung (BMBF) herangezogen werden, können wie folgt differenziert werden:

- Bei den Prüfkriterien handelt es sich um solche, die für staatliche Maßnahmen von besonderer Bedeutung sind wie etwa Neuheit oder Marktnähe.

- Ermittlungskriterien bieten eine Basis zur näheren Einschätzung des Technologiestandes und seiner Perspektiven wie etwa Anwendungspotenzial.

- Rahmenkriterien schließlich beziehen sich auf übergeordnete Ziele wie die von einer Technologie erwarteten Beiträge hinsichtlich ökonomischer, politischer, ökologischer und sozialer Dimensionen.

Auf Basis dieser Bewertung ist es möglich, fundierte strategische Empfehlungen und geeignete innovationsbegleitende Maßnahmen zu formulieren.

Dritter Schritt, wiederum im Falle des Beispiels der Früherkennung für das BMBF, ist das Zusammenführen potentieller Anwender aus der Wirt-

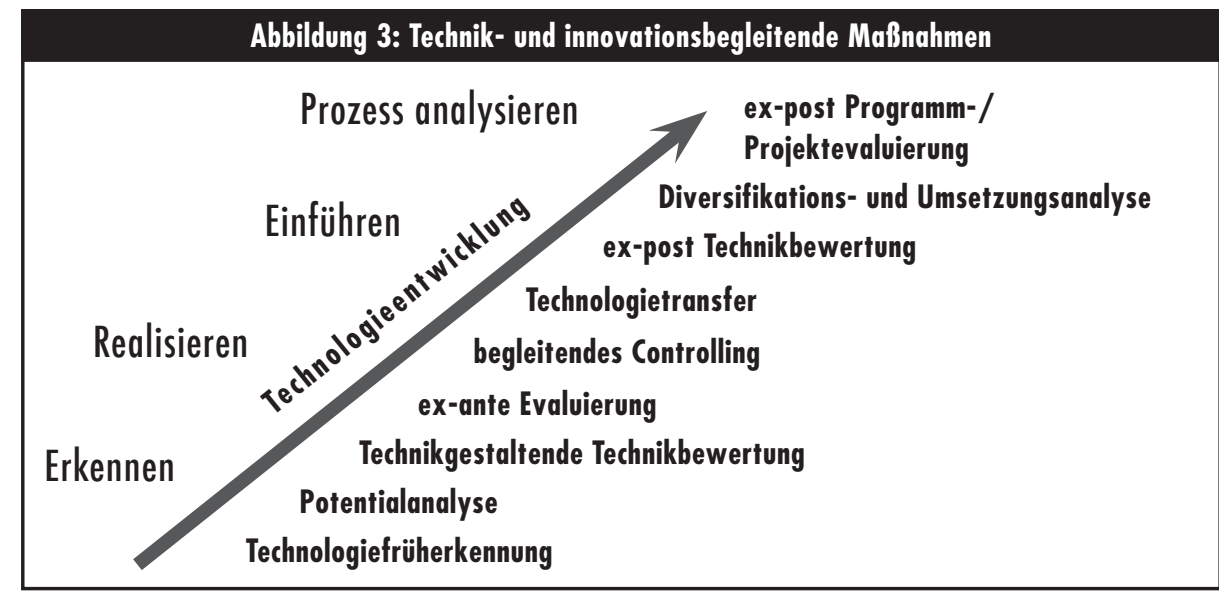

Quelle: VDI

schaft mit Experten, die in dem identifizierten Gebiet arbeiten. Diese Teilaufgabe wird als Umsetzung bezeichnet. Durch Workshops und Veranstaltungen werden folgende Ziele anvisiert:

- Potentielle Anwender konkretisieren ihr Interesse.

- Vorbereitung strategischer Entscheidungen zur Unterstützung des neuen Technologiegebiets.

- Beginn einer von Fachwissenschaftlern geführten, interdisziplinären Debatte.

Abbildung 2 bietet als Beispiel eine Bewertungstabelle, wie sie im Rahmen einer Technologieanalyse zur Nanotechnologie publiziert wurde. Für verschiedene Teilgebiete der Nanotechnologie wurden hier im Rahmen der Technologiefrüherkennung gewonnene Ergebnisse radikal kondensiert und in einer Übersichtsform zusammengetragen. Derartige Übersichten sind immer vor dem Hintergrund der sie begleitenden Studien zu betrachten, ein Punkt, der leider immer wieder übersehen wird und zu unzulässigen Verkürzungen führt.
Abbildung 3 verdeutlicht, dass Technologiefrüherkennung nicht die einzige technikbegleitende Maßnahme zur Beschleunigung und Umsetzung zukunftsträchtiger Technologien ist. Eine Auswahl wichtiger Maßnahmen wird hier zeitlich den verschiedenen Phasen der Technologieentwicklung zugeordnet.

Technologiefrüherkennung darf nicht als isolierte technikbegleitende Maßnahme betrachtet werden. Ein unverzahntes Platzieren einzelner Maßnahmen unterbindet die effiziente Gestaltung der Maßnahmen. Sich partiell widersprechende Einsichten beeinflussen den Innovationsprozess stets nur tendenziell in die gewünschte Richtung. Erst ein Verzahnen der Begleitmaßnahmen sichert die gewünschte effektive Unterstïtzung des Innovationsprozesses. Diese Verknüpfung innovationsbegleitender Maßnahmen im Sinne einer ganzheitlicheren Betrachtung führt zu einem 'Integrierten Technologie und Innovationsmanagement', dass die für die Begleitmaßnahmen eingesetzten Ressourcen effizient nutzt

\section{Abbildung 2: Auszug aus einer Technologieanalyse}

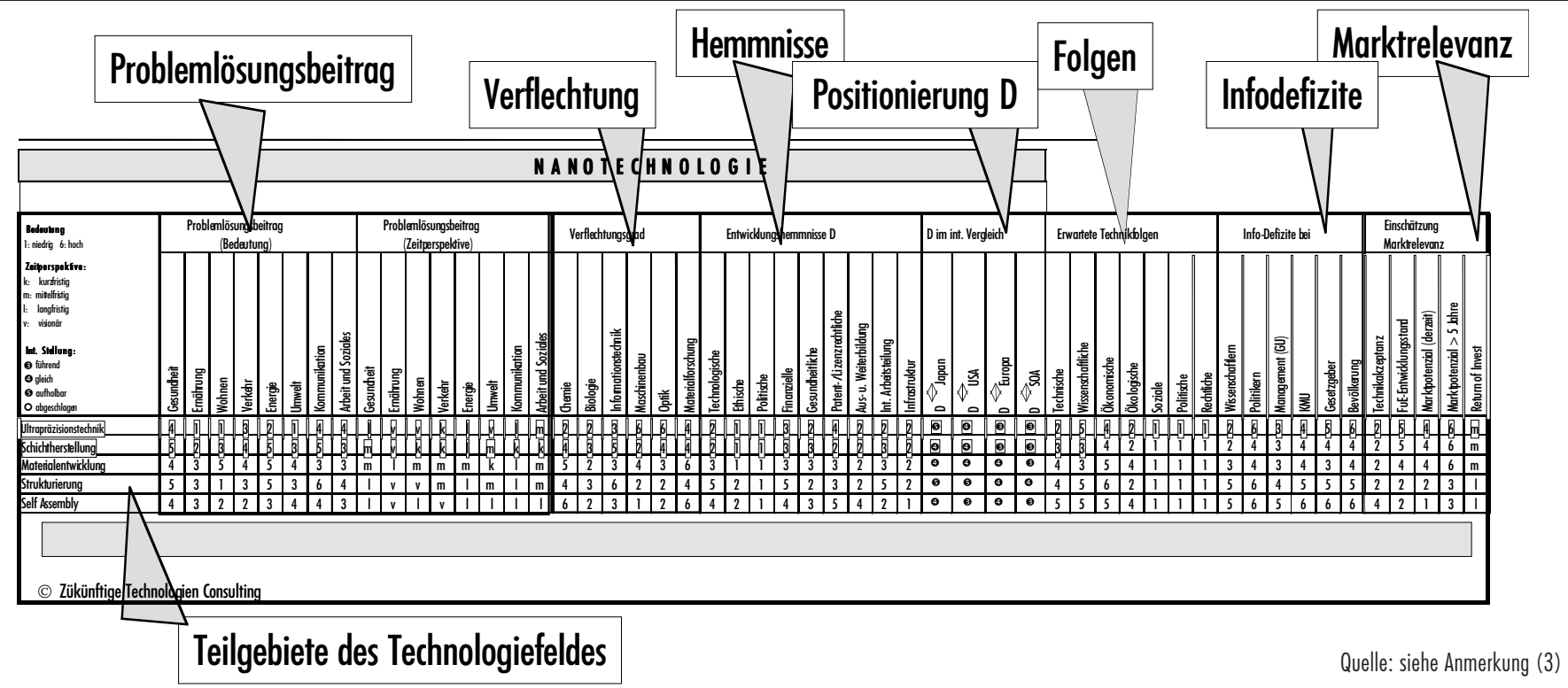


Grafik 4: Entwicklung von Forschungs- und Technologiepolitiken

\begin{tabular}{|c|c|c|c|}
\hline Zeitraum & 1950-75 & 1975-95 & 2000 und danach \\
\hline Hauptziel & politisch & wirtschaftlich & Gesellschaftlich \\
\hline Fokus & militärische Sicherheit & $\begin{array}{l}\text { industrielle Wett- } \\
\text { bewerbsfähigkeit }\end{array}$ & $\begin{array}{l}\text { Beschäftigung und } \\
\text { Lebensqualität }\end{array}$ \\
\hline Perpektive & national & international & weltweit \\
\hline Federführende Ministerien & $\begin{array}{l}\text { Verteidigung, Bildung und } \\
\text { Forschung }\end{array}$ & Bildung und Forschung & interministerielle Koordination \\
\hline Wichtigste Technologien & $\begin{array}{l}\text { Nukleartechn., Luft- und } \\
\text { Raumfahrt }\end{array}$ & $\begin{array}{l}\text { Elektronik, Informatik } \\
\text { Telekomunikation }\end{array}$ & $\begin{array}{l}\text { Mischwissenschaften und } \\
\text { technologien, Kombination je } \\
\text { nach Problemstellung }\end{array}$ \\
\hline Durchführung & $\begin{array}{l}\text { staatliche Forschungs- } \\
\text { einrichtungen }\end{array}$ & $\begin{array}{l}\text { Förder- und Kooperations- } \\
\text { programme }\end{array}$ & $\begin{array}{l}\text { Task Forces, interdisziplinäre } \\
\text { Programme und Projekte }\end{array}$ \\
\hline $\begin{array}{l}\text { Vorherrschende } \\
\text { Auswahlkriterien }\end{array}$ & $\begin{array}{l}\text { wissenschaftlicher } \\
\text { Höchststand }\end{array}$ & $\begin{array}{l}\text { wissenschaftlicher } \\
\text { Höchststand und Beitag zur } \\
\text { Wettbewerbsfähigkeit }\end{array}$ & $\begin{array}{l}\text { Beitrag zu den Bedürnissen } \\
\text { der Gesellschaft und der } \\
\text { Industrie }\end{array}$ \\
\hline
\end{tabular}

und die Potentiale einzelner Maßnahmen voll entfaltet (4). Zwar bietet Technologiefrüherkennung ein Instrument, das bereits bei der Identifikation zukunftsrelevanter Technologien, also frühzeitig im Innovationszyklus, die Berücksichtigung sozio-ökonomischer wie auch nachhaltigkeits-bezogener Aspekte ermöglicht, erst aber ein integriertes Technologie- und Innovationsmanagement vermag sicher zu stellen, dass vor allem nachhaltigkeits-bezogene Aspekte im weiteren Innovationsprozess kontinuierlich einbezogen werden.

Das Bedürfnis, künftige Entwicklungen und Trends mit gewisser Sicherheit zu antizipieren, wächst in dem Maße, wie sich der globale Wettbewerb verschärft. Sowohl im wissenschaftlich-technischen als auch im sozio-ökonomischen Bereich sind in Deutschland enorme Wissenspotentiale vorhanden. Innerhalb Europas besitzt Deutschland wohl die grundsätzlich differenzierteste Landschaft zukunftsbefasster Fachszenen für Technologiefrüherkennung, Technikfolgenabschätzung oder Foresight. Jede der Fachszenen bietet zukunftsbezogene Antworten zu speziellen Ausgangsfragestellungen und übergeordneten Betrachtungsperspektiven. Langfristig sichert diese Pluralität unterschiedlicher Ausgangsfragen und verschiedenartigster Erhebungsverfahren den notwendigen Bodensatz an Kreativität und originären Ideen (5).

Staatliche Forschungs- und Innovationspolitiken werden zunehmend dieser umfassenden Herausforderung gerecht. Abbildung 4 skizziert den Wandel staatlicher Forschungs- und Innovationspolitiken von politischen über wirtschaftliche zu gesellschaftlichen Hauptzielen. Zugleich erweitert sich die Perspektive von einer nationalen über eine transnational gedachte hin zu einer weltweiten. Vor dem Hintergrund der treibenden Kräfte zu Beginn des 21. Jahrhunderts, Globalisierung
Eine besondere Bedeutung im Zusammenhang mit zukunftsrelevanten Technologien muss die Reflexion über Nachhaltigkeit spielen. Dies gilt für die Technologiefrüherkennung, die sich zu einem sehr frühen Zeitpunkt des Innovationsprozesses damit auseinandersetzt, ebenso wie für die Diskussion um künftige Visionen unserer Gesellschaft und der dafür erforderlichen Qualitäten von Technologien. Nicht vergessen werden darf, dass sich das Konzept der Nachhaltigkeit in Konkurrenz zu anderen - je nach Sicht mehr oder weniger konfligierenden - Anforderungen befindet und sich wieder und wieder im gesamten Innovationsprozess Gehör verschaffen muss.

\section{Anmerkungen}

der Finanzmärkte, Revolution der Lebenswissenschaften, Nachhaltigkeit, demografische und soziale Entwicklung, sind verstärkt partizipativ getragene Prozesse erforderlich (6). Diese Prozesse sollen eine breite und intensive öffentliche Auseinandersetzung mit unserer gesellschaftlichen und technologischen Zukunft ermöglichen.

Um gesellschaftliche Wünsche und Trends ebenso wie längerfristige sozio-ökonomische Prognosen einzubeziehen, werden gegenwärtig gern sogenannte Foresight-Prozesse - wie zum Beispiel der deutsche FUTUR-Prozess - herangezogen (8). Ihr Ziel ist eine Vision, die gesellschaftlichen und wirtschaftlichen Bedarf sowie technisch-wissenschaftliches Angebot zusammenführt, und diese Vision über einen partizipativen Prozess erlangt (9). Ein Foresight-Prozess darf aber nicht bei der integrierenden Aufbereitung vorhandenen Wissens zu konkreten Fragenkomplexen stehen bleiben. Erforderlich ist darüber hinaus eine reflektierende Kommunikation dieser Visionen durch gesellschaftliche Kräfte und Institutionen. Von besonderer Bedeutung ist die öffentliche Verfügbarkeit der Ergebnisse beider Schritte, d. h. des Integrations- und Kommunikationsprozesses. Damit ergeben sich als grundsätzliche Ziele eines derartigen nationalen Foresight-Prozesses zusammenfassend:

- Zusammenstellen und Zusammenführen sozioökonomischer Trends und technisch-wissenschaftlicher Entwicklungslinien (Integration vorhandenen Wissens)

- Reflexion der Trends durch organisierte Repräsentanten aus Gesellschaft, Politik, Wirtschaft und Wissenschaft (Kommunikation durch gesellschaftliche Akteure)

- Begleitende und mitgestaltende öffentliche Diskussion (Partizipation)
(1) Revermann, H./ Sonntag, P.: Schlüsseltechnologien; Turbulenter Wandel der Industrie durch innovative Dynamik. Berlin, Offenbach 1987.

(2) Vgl. Bundesministerium für Forschung und Technologie (Hg.): Deutscher Delphibericht zur Entwicklung von Wissenschaft und Technik. Bonn 1992; Fhg-ISI (Hg.): Delphi '98 Umfrage - Studie zur globalen Entwicklung von Wissenschaft und Technik. Karlsruhe 1998; Grupp, H.: Technologie am Beginn des 21. Jahrhunderts. FhG-ISI, Heidelberg 1992; Revermann, H./ Sonntag, P. a.a.0.

(3) Bachmann, G.: Analyse und Bewertung zukünftiger Technologien. Innovationsschub aus dem Nanokosmos. VDITechnologiezentrum, Düsseldorf 1998.

(4) Zweck, A.: Zur Gestaltung technischen Wandels - Integriertes Technologie- und Innovationsmanagement (ITIM) begleitet Innovationen ganzheitlich. In: Wissenschaftsmanagement 2, 2003. S. 25-32.

(5) Zweck, A.: Three perspectives for one future in economy and society. In: Futures Research Quarterly, vol. 18, no. 1, Spring 2002. S. 55-66.

(6) Malanowski, N./ Seiler, P.: Partizipative Innovationsund Technikanalyse - Warum, mit welchem Erfolg und auf welchen Wegen? In Development and Perspectives 1, 2003. (Im Druck).

(7) Caracostas, P./ Muldur, U.: Die Gesellschaft letzte Grenze. Eine europäische Vision der Forschungs- und Innovationspolitik im XXI. Jahrhundert. GDXII, Brüssel 1998. (8) Dietz, V.: FUTUR- der deutsche Forschungsdialog. In: Development and Perspectives 1, 2002. S. 3-24

(9) Zweck, A./ Braun, M.: Foresight - Ein Blick in die Zukunft zwischen Anspruch und Partizipation. In: Development and Perspectives 1, 2002. S. 47-65.

\section{Der Autor}

Dr. Dr. Axel Zweck leitet die Zukünftige TechnoloTechnologiezentrum (VDI-TZ-ZTC).

Kontakt: VDI-TZ-ZTC, Graf-Recke-Str. 84, 40239 Düsseldorf. Tel. 0211-6214-572, Fax -484, E-Mail: zweck@vdi.de gien Consulting des Vereins Deutscher Ingenieure 
(c) 20I0 Authors; licensee IÖW and oekom verlag. This is an article distributed under the terms of the Creative Commons Attribution Non-Commercial No Derivates License (http://creativecommons.org/licenses/by-nc-nd/3.o/), which permits unrestricted use, distribution, and reproduction in any medium, provided the original work is properly cited. 